\title{
An Epidemic Model for the Propagation of Leptospirosis Outbreaks
}

\author{
Ariel Félix Gualtieri and Juan Pedro Hecht \\ Universidad de Buenos Aires, Facultad de Odontología, Cátedra de Biofísica y Bioestadística, Buenos Aires 1053, Argentina
}

\begin{abstract}
Leptospirosis is a disease of worldwide distribution caused by spirochetes of the genus Leptospira. Rodents have been recognized as the most important and most widely distributed reservoirs of leptospirosis. In different regions of the world, there are outbreaks of leptospirosis that have a seasonal distribution and coincide with the rainy months. One way to study the spread of epidemics is through mathematical models. In the present work, the objective of the authors was to develop and explore a model of leptospirosis outbreak, combining the dynamics of propagation in human population with the dynamics of transmission from rodent reservoirs to humans. The authors designed a deterministic model based on differential equations. The dynamics of the model were explored through computational simulations. The typical evolution of prevalence observed in the model, qualitatively coincides with the dynamics of actual leptospirosis outbreaks. In the model, the peak prevalence was reduced when the transmission parameter and the number of rodents decreased, and when the lepospira decay rate increased. These results coincide with what was expected. The present development could be considered as a starting point for the generation of a more complex design.
\end{abstract}

Key words: Leptospirosis, epidemic model, simulation, public health.

\section{Introduction}

Epidemic models are formal designs that allow capturing the behavior of the propagation of infectious diseases. They achieve this goal through the mathematical representation of processes that regulate the dynamics of outbreaks. In this way, they can provide trends regarding the results of different health interventions and the influence of certain factors of interest on the problem addressed [1-4].

The theoretical approach of the epidemiological models is based on the two elements mentioned below: (1) the population is divided into categories according to the different stages considered within the disease under study and (2) the temporal evolution of the number of individuals in each of these categories is formulated mathematically. For example, in the classic SIR (susceptible-infected-recovered) scheme,

Corresponding author: Ariel Félix Gualtieri, Ph.D., research fields: applied mathematics, epidemiology, biostatistics and public health.

Co-author: Juan Pedro Hecht, Ph.D., research fields: applied mathematics, biological complexity and biostatistics. the population is divided into three successive categories: susceptible (S), infected (I) and recovered with immunity (R). According to this grouping, transitions S-I (infection) and I-R (recovery) are defined mathematically as a function of time [5, 6].

At present, epidemiological models based on approaches of different complexity coexist. In the literature can be found from classic designs represented by systems of differential equations [7-9], to the most advanced computational simulation developments [10-12]. Differential equations models, such as the one developed in the present paper, have a simple construction and can be explored by unsophisticated computational means. The purposes for which the epidemiological mathematical models are developed are many and varied [1]. There are models that are developed to be used in real time during a particular epidemic. Others, such as the one presented in the present work, are developed for the general understanding of the dynamics of transmission through a qualitative analysis.

Despite the continuous and growing development 
of research in epidemiological models, not all diseases have had the same level of attention. Lloyd-Smith et al. [13], in their review of models of zoonotic diseases, show a remarkable imbalance. Among the diseases that may involve some step of transmission from species of vertebrate reservoirs to man, more than half of the studies collected by these authors correspond to rabies, influenza or severe acute respiratory syndrome. Some diseases of important sanitary interest have been relegated. Leptospirosis is one of them.

Leptospirosis is a disease of worldwide distribution caused by spirochetes of the genus Leptospira $[14,15]$. It is an important emerging public health problem [16-18]. The disease affects several animals, including rats and other rodents, cattle and dogs [19, 20]. The man acquires the disease by exposure to urine from infected animal reservoirs, which eliminate spirochetes through urinary pathway [21]. Exposure can be direct, or indirect, by contamination of soil and water $[14,16]$. Transmission between humans is considered unimportant [14, 22]. The disease produces in man various symptoms, which may include fever, headache, myalgia, jaundice, acute renal failure and conjunctival disease $[23,24]$. On average, the case-fatality rate would be around 7\% [25].

Rodents have been recognized as the most important and most widely distributed reservoirs of leptospirosis [26-28]. A known risk factor for outbreaks of leptospirosis includes the prevalence in communities of rodents neighboring human populations [21]. High percentages of leptospirosis prevalence in Rattus norvegicus have been found in several cities, such as Tokyo (17\%) [29], Baltimore (90.4\%) [30], Copenhagen (48-89\%) [31] and Gran Buenos Aires (45.8\%) [32]. The occurrence of Leptospira infection is more frequent in humid and warm climates [33]. The disease is mainly located in the Caribbean, Central and South America, the Indian subcontinent, Southeast Asia, Oceania and, to a lesser extent, in Eastern Europe [34]. Leptospirosis can have two conditions: endemic and epidemic [35, 36]. In epidemic dynamics, which will be represented through the model developed in the present work, there are outbreaks that occur after the rains and floods, where water serves as a vehicle for infection [37-40].

Previously, leptospirosis propagation models have been developed (for example, Refs. [28, 41-46]). In the present work, the objective of the authors was to develop and explore a model of leptospirosis outbreak, combining the dynamics of propagation in human population with the dynamics of transmission from rodent reservoirs to humans.

\section{Materials and Methods}

\subsection{The Model}

A deterministic model based on differential equations was developed to simulate the propagation of a seasonal outbreak of leptospirosis. The model contemplates a community formed by humans and rodent reservoirs. Rodents release spirochetes that reach humans indirectly through flood waters. The model is represented by the following system of equations:

$$
\begin{aligned}
\frac{\mathrm{d} S}{\mathrm{dt}} & =-\mathrm{aSL}, \\
\frac{\mathrm{dI}}{\mathrm{dt}} & =\mathrm{aSL}-\mathrm{bI}-\mathrm{cI}, \\
\frac{\mathrm{dR}}{\mathrm{dt}} & =\mathrm{bI}, \\
\frac{\mathrm{dL}}{\mathrm{dt}} & =\mathrm{eM}-\mathrm{fL} .
\end{aligned}
$$

A SIR scheme was considered for humans, so people were divided into susceptible $(S)$, infected $(I)$ and recovered $(R)$ individuals. In this way, $S(t), I(t)$ and $R(t)$ are the number of susceptible, infected and recovered individuals at time $t$, respectively. $L(t)$ is the number of spirochetes in flood waters at time $t$. Humans become infected when they come into contact with spirochetes in flood waters. It is assumed that the net rate at which infection occurs is proportional to the number of encounters between susceptible individuals 
and spirochetes, $a S L$, where $a$ is the transmission parameter. Infected individuals recover and die with rates $b$ and $c$, respectively. The number of spirochetes released depends on the population size of the infected reservoirs, $M$. Spirochetes are released with an $e$ rate and decay with a $f$ rate, respectively.

\subsection{Numerical Simulations}

The dynamics of the model were explored through computational simulations. Specifically, the evolution of prevalence as a function of time was represented. The recovery rate $b$ was based on Ref. [14], and the lethality rate $c$ on Ref. [47]. The values of parameters $e$ and $f$ were in the order of the estimates mentioned in Ref. [28]. An arbitrary value of $a$ was set because it was not found in the literature. In theoretical epidemiological models it is common to use arbitrary values, mainly when a qualitative analysis is intended (see, for example, Ref. [48]).

Arbitrary values of the initial conditions were set, but a human:infected rodent ratio of 1:3 was chosen, according to the information documented in several works [32, 49-51]. Time and parameters are expressed in day and 1/day, respectively. A benchmark case was established, where the following values were set for the initial conditions and parameters: $S(0)=100, I(0)$ $=0, R(0)=0, L(0)=0, a=10^{-8}, b=0.14, c=0.014, e$ $=10^{3}, f=0.10, M=300$, with the parameters $a, b, c, e$ and $f$ expressed in $1 /$ day.

The influence of parameters $a, M$ and $f$ on the evolution of prevalence was explored. The simulations were implemented in the program Dynamics Solver 1.98 [52]. The numerical results obtained in the simulations were represented graphically using the program Infostat 2018 [53].

\section{Results}

The typical evolution of the prevalence predicted by the model, using the values of the benchmark case, is represented in Fig. 1. It is observed that the prevalence grows exponentially to a peak and then decreases.

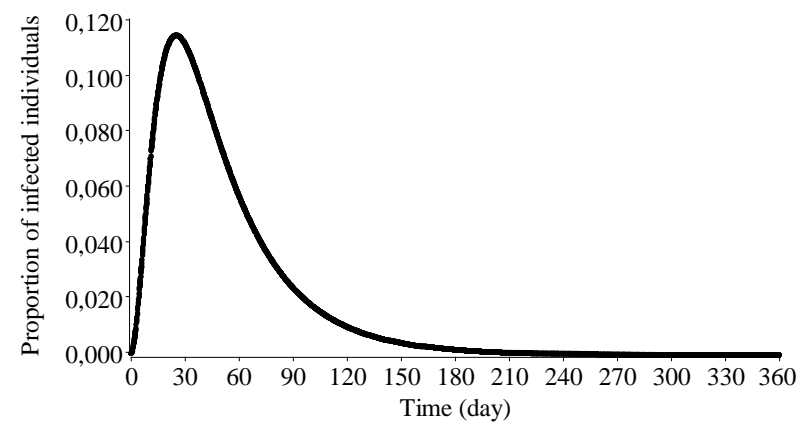

Fig. 1 Evolution of the prevalence in the benchmark case. Initial conditions and parameters: $S(0)=100, I(0)=0, R(0)$ $=0, L(0)=0, a=10^{-8}, b=0.14, c=0.014, e=10^{3}, f=0.10, M$ $=300(a, b, c, e$ and $f$ expressed in 1/day).

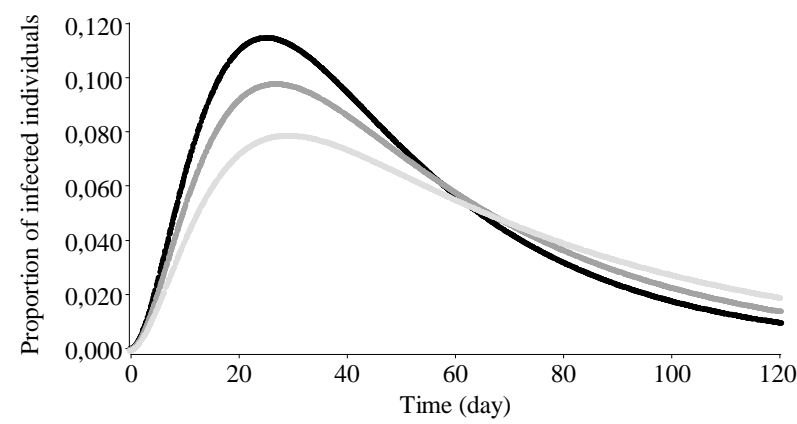

Fig. 2 Influence of the transmission parameter $a$ on the evolution of the prevalence. Three values were evaluated (1/day): $a_{1}=10^{-8}$ (black), $a_{2}=8 \times 10^{-9}$ (dark grey), $a_{3}=6 \times$ $10^{-9}$ (light grey). The rest of the conditions were maintained as in the benchmark case.

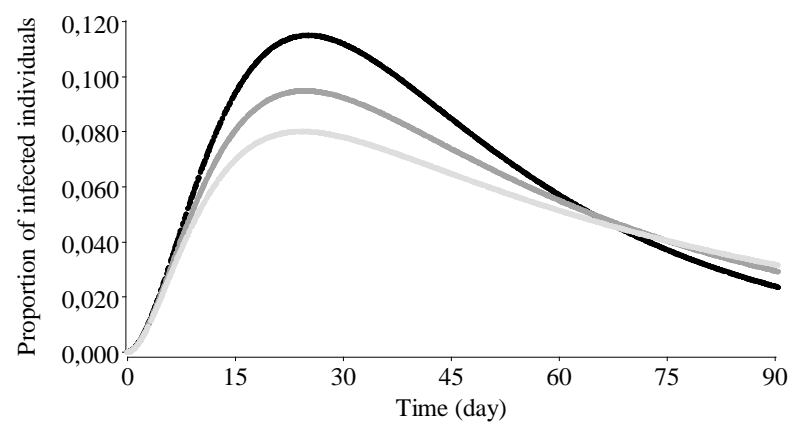

Fig. 3 Influence of lepospiral decay parameter $f$ on the evolution of prevalence. Three values were evaluated (1/day): $f_{1}=0.10$ (black), $f_{2}=0.14$ (dark grey), $f_{3}=0.18$ (light grey). The rest of the conditions were maintained as in the benchmark case.

In order to study the influence of the transmission parameter $a$ on the evolution of the system, three values (1/day) were compared: $a_{1}=10^{-8}$ (benchmark), $a_{2}=8 \times 10^{-9}, a_{3}=6 \times 10^{-9}$. The reduction of $a$ caused a drop in peak prevalence (Fig. 2). With the purpose 


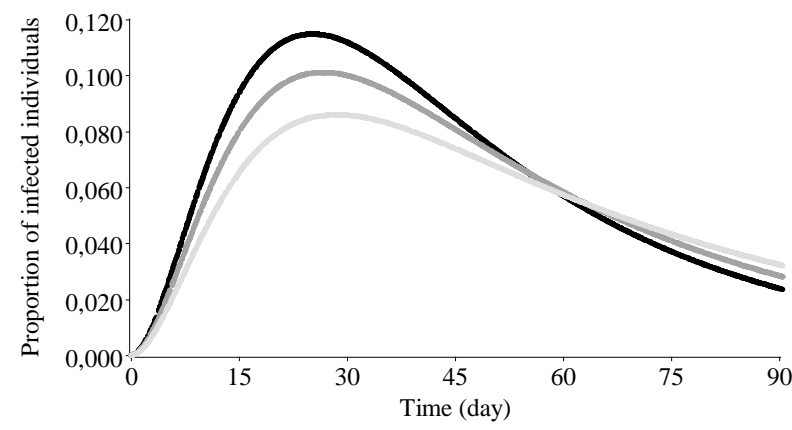

Fig. 4 Influence of the population size of rodents $M$ on the evolution of prevalence. Three values were evaluated: $M_{1}=$ 300 (black), $M_{2}=250$ (dark grey), $M_{3}=200$ (light grey). The rest of the conditions were maintained as in the reference case.

of evaluating the behavior of the system as a function of the leptospiral decay parameter $f$, the following values were compared (1/day): $f_{1}=0.10$ (benchmark), $f_{2}=0.14, f_{3}=0.18$. The increase in $f$ produced a reduction in peak prevalence (Fig. 3). When different rodent population size values were compared $\left(M_{1}=\right.$ 300, benchmark; $M_{2}=250$ and $M_{3}=200$ ), it was observed that the decrease in rodents led to a decline in peak prevalence (Fig. 4).

\section{Discussion}

In different regions of the world, there are outbreaks of leptospirosis that have a seasonal distribution and coincide with the rainy months [14, 21, 33, 54-56]. For example, in Argentina, a national study conducted between 1999 and 2005 found that the greatest incidence in humans occurs between mid-summer and late autumn, coinciding with hot and rainy weather [23]. In the present work, the authors have tried to simulate qualitatively the propagation of leptospirosis in such scenarios.

Holt et al. [28] developed a model for the spread of leptospirosis in one species of African rodent. Their model includes the release of leptospires into the environment and infection in reservoirs, but does not include humans. In the present paper, the authors relied on the development of Holt et al. [28] to model the release of leptospires into the environment, but represented the dynamics of infection in humans. The model presented also includes elements from the works of Khan et al. [45] and Shukla et al. [57].

The typical evolution of prevalence observed in the model developed by the authors, qualitatively coincides with the dynamics of actual leptospirosis outbreaks (see, for example, Refs. [23] and [38]), where the number of cases increases to a peak and then drops to very low values.

When the evolution of the model as a function of the transmission parameter $a$ was studied, the simulations showed that a reduction of $a$ produced a reduction of the peak prevalence. Parameter $a$ is related to the transmission probability when susceptible humans are exposed to leptospira in flood waters. The reduction of parameter $a$ could represent an increase in the application of preventive measures, such as wearing appropriate protective clothing (waterproof boots, for example) when walking in a flooded area [17, 22]. In this way, the results observed in the simulations would coincide with what was expected.

During a flood, the concentration of leptospira in flooded areas may depend inversely on the distance to places where rodent droppings are highly concentrated, such as dumps [37]. Parameter $f$ of the model developed in this paper, regulates the decay of leptospira in the environment. In this way, the increase of $f$ could simulate the effect of an increase in the distance to a dump. In accordance with this approach, the simulations carried out show that an increase of $f$ produces a reduction of the prevalence peak.

Reducing rodent populations is also an action that could be taken to control leptospirosis [22, 26, 55]. Congruently, when the model simulated a decrease in rodent numbers, a drop in human prevalence was observed.

\section{Conclusion}

The authors developed an original mathematical model that, through a relatively low number of parameters, qualitatively simulates the evolution of a 
leptospirosis outbreak and also allows exploring some variables related to the behavior of this phenomenon. Future work could be oriented to formally verify the results provided by the model. Likewise, the present development could be considered as a starting point for the generation of a more complex design.

\section{Acknowledgments}

The authors gratefully acknowledge Universidad de Buenos Aires for providing financial support (grant numbers UBACyT 20020110200037BA, UBACyT 20020130100417BA).

\section{References}

[1] Keeling, M. J., and Rohani, P. 2008. Modeling Infectious Diseases in Humans and Animals. Princeton: Princeton University Press.

[2] Huppert, A., and Katriel, G. 2013. "Mathematical Modelling and Prediction in Infectious Disease Epidemiology.” Clinical Microbiology and Infection 19 (11): 999-1005.

[3] Brauer, F. 2017. "Mathematical Epidemiology: Past, Present, and Future.” Infectious Disease Modelling 2 (2): 113-27.

[4] Walters, C. E., Meslé, M. M. I., and Hall, I. M. 2018. "Modelling the Global Spread of Diseases: A Review of Current Practice and Capability.” Epidemics (25): 1-8.

[5] Brauer, F. 2008. "Compartmental Models in Epidemiology.” In Mathematical Epidemiology, edited by Brauer, F., van den Driessche, P., and Wu, J. Berlin Heidelberg: Springer-Verlag.

[6] Martcheva, M. 2015. An Introduction to Mathematical Epidemiology. Vol. 61. New York: Springer.

[7] Khalid, M., and Khan, F. S. 2016. "Stability Analysis of Deterministic Mathematical Model for Zika Virus.” British Journal of Mathematics \& Computer Science 19 (4): 1-10.

[8] Ngwa, G. A., and Teboh-Ewungkem, M. I. 2016. “A Mathematical Model with Quarantine States for the Dynamics of Ebola Virus Disease in Human Populations.” Computational and Mathematical Methods in Medicine Art. ID 9352725: 29.

[9] Osman, S., Makinde, O. D., and Theuri, D. M. 2018. "Mathematical Modelling of Transmission Dynamics of Anthrax in Human and Animal Population." Mathematical Theory and Modelling 8 (6): 47-67.

[10] Gualtieri, A. F., and Hecht, J. P. 2016. "Stochastic Lattice Gas Cellular Automata Model for Epidemics.” Journal of Life Sciences 10 (2): 77-84.
[11] Sharma, N., and Gupta, A. K. 2017. "Impact of Time Delay on the Dynamics of SEIR Epidemic Model Using Cellular Automata.” Physica A: Statistical Mechanics and its Applications 471: 114-25.

[12] Brown, G. D., Porter, A. T., Oleson, J. J., and Hinman, J. A. 2018. "Approximate Bayesian Computation for Spatial SEIR (S) Epidemic Models.” Spatial and Spatio-Temporal Epidemiology 24: 27-37.

[13] Lloyd-Smith, J. O., George, D., Pepin, K. M., Pitzer, V. E., Pulliam, J. R., Dobson, A. P., ... and Grenfell, B. T. 2009. "Epidemic Dynamics at the Human-Animal Interface.” Science 326 (5958): 1362-7.

[14] Bharti, A. R., Nally, J. E., Ricaldi, J. N., Matthias, M. A., Diaz, M. M., Lovett, M. A., ... and Vinetz, J. M. 2003. "Leptospirosis: A Zoonotic Disease of Global Importance." The Lancet Infectious Diseases 3 (12): 757-71.

[15] Evangelista, K. V., and Coburn, J. 2010. "Leptospira as an Emerging Pathogen: A Review of Its Biology, Pathogenesis and Host Immune Responses.” Future Microbiology 5 (9): 1413-25.

[16] Vijayachari, P., Sugunan, A. P., and Shriram, A. N. 2008. "Leptospirosis: An Emerging Global Public Health Problem.” Journal of Biosciences 33 (4): 557-69.

[17] Hartskeerl, R. A., Collares-Pereira, M., and Ellis, W. A. 2011. "Emergence, Control and Re-emerging Leptospirosis: Dynamics of Infection in the Changing World." Clinical Microbiology and Infection 17 (4): 494-501.

[18] Torgerson, P. R., Hagan, J. E., Costa, F., Calcagno, J., Kane, M., Martinez-Silveira, M. S., ... and Abela-Ridder, B. 2015. "Global Burden of Leptospirosis: Estimated in Terms of Disability Adjusted Life Years.” PLoS Neglected Tropical Diseases 9 (10): e0004122.

[19] Barragan, V., Nieto, N., Keim, P., and Pearson, T. 2017. "Meta-analysis to Estimate the Load of Leptospira Excreted in Urine: Beyond Rats as Important Sources of Transmission in Low-income Rural Communities.” BMC Research Notes 10 (1): 71.

[20] Lau, C. L., Smythe, L. D., Craig, S. B., and Weinstein, P. 2010. "Climate Change, Flooding, Urbanisation and Leptospirosis: Fuelling the Fire?" Transactions of the Royal Society of Tropical Medicine and Hygiene 104 (10): 631-8.

[21] Socolovschi, C., Angelakis, E., Renvoisé, A., Fournier, P. E., Marie, J. L., Davoust, B., ... and Raoult, D. 2011. "Strikes, Flooding, Rats, and Leptospirosis in Marseille, France.” International Journal of Infectious Diseases 15 (10): e710-5.

[22] Lim, V. K. 2011. "Leptospirosis: A Re-emerging Infection.” The Malaysian Journal of Pathology 33 (1): $1-5$. 
[23] Vanasco, N. B., Schmeling, M. F., Lottersberger, J., Costa, F., Ko, A. I., and Tarabla, H. D. 2008. "Clinical Characteristics and Risk Factors of Human Leptospirosis in Argentina (1999-2005).” Acta Tropica 107 (3): 255-8.

[24] Haake, D. A., and Levett, P. N. 2015. "Leptospirosis in Humans.” In Leptospira and Leptospirosis, edited by Adler, B. Berlin Heidelberg: Springer-Verlag.

[25] Costa, F., Hagan, J. E., Calcagno, J., Kane, M., Torgerson, P., Martinez-Silveira, M. S., ... and Ko, A. I. 2015. "Global Morbidity and Mortality of Leptospirosis: A Systematic Review.” PLoS Neglected Tropical Diseases 9 (9): e0003898.

[26] WHO (World Health Organization). 2003. Human Leptospirosis: Guidance for Diagnosis, Surveillance and Control. Geneva: WHO.

[27] Costa, F., Wunder Jr, E. A., De Oliveira, D., Bisht, V., Rodrigues, G., Reis, M. G., ... and Childs, J. E. 2015. "Patterns in Leptospira Shedding in Norway Rats (Rattus norvegicus) from Brazilian Slum Communities at High Risk of Disease Transmission.” PLoS Neglected Tropical Diseases 9 (6): e0003819.

[28] Holt, J., Davis, S., and Leirs, H. 2006. “A Model of Leptospirosis Infection in an African Rodent to Determine Risk to Humans: Seasonal Fluctuations and the Impact of Rodent Control." Acta Tropica 99 (2-3): 218-225.

[29] Koizumi, N., Muto, M., Tanikawa, T., Mizutani, H., Sohmura, Y., Hayashi, E., ... and Watanabe, H. 2009. "Human Leptospirosis Cases and the Prevalence of Rats Harbouring Leptospira Interrogans in Urban Areas of Tokyo, Japan.” Journal of Medical Microbiology 58 (9): 1227-30.

[30] Vinetz, J. M., Glass, G. E., Flexner, C. E., Mueller, P., and Kaslow, D. C. 1996. "Sporadic Urban Leptospirosis.” Annals of Internal Medicine 125 (10): 794-8.

[31] Krøjgaard, L. H., Villumsen, S., Markussen, M. D. K., Jensen, J. S., Leirs, H., and Heiberg, A. C. 2009. "High Prevalence of Leptospira spp. in Sewer Rats (Rattus norvegicus).” Epidemiology \& Infection 137 (11): 1586-1592.

[32] Arango, J., Cittadino, E., Agostini, A., Dorta de Mazzonelli, G., Alvarez, C., Colusi, M., ... and Kravetz, F. 2001. "Prevalencia de leptospiras en Rattus rattus y Rattus norvegicus en el Gran Buenos Aires, Argentina.” Ecología Austral 11: 25-30. [in Spanish].

[33] Levett, P. N. 2001. "Leptospirosis.” Clinical Microbiology Reviews 14 (2): 296-326.

[34] Pappas, G., and Cascio, A. 2006. "Optimal Treatment of Leptospirosis: Queries and Projections.” International Journal of Antimicrobial Agents 28 (6): 491-6.

[35] Johnson, M. A., Smith, H., Joseph, P., Gilman, R. H., Bautista, C. T., Campos, K. J., ... and Calderon, M. M.
2004. "Environmental Exposure and Leptospirosis, Peru." Emerging Infectious Diseases 10 (6): 1016-22.

[36] Schneider, M. C., Leonel, D. G., Hamrick, P. N., Caldas, E. P. D., Velásquez, R. T., Paez, F. A. M., ... and Aldighieri, S. 2017. "Leptospirosis in Latin America: Exploring the First Set of Regional Data." Revista Panamericana de Salud Pública 41: e81.

[37] Barcellos, C., and Sabroza, P. C. 2001. "The Place behind the Case: Leptospirosis Risks and Associated Environmental Conditions in a Flood-related Outbreak in Rio de Janeiro.” Cadernos de Saúde Pública 17: S59-S67.

[38] Agampodi, S. B., Dahanayaka, N. J., Bandaranayaka, A. K., Perera, M., Priyankara, S., Weerawansa, P., ... and Vinetz, J. M. 2014. "Regional Differences of Leptospirosis in Sri Lanka: Observations from a Flood-associated Outbreak in 2011." PLoS Neglected Tropical Diseases 8 (1): e2626.

[39] Dechet, A. M., Parsons, M., Rambaran, M., Mohamed-Rambaran, P., Florendo-Cumbermack, A., Persaud, S., ... and Paddock, C. D. 2012. "Leptospirosis Outbreak Following Severe Flooding: A Rapid Assessment and Mass Prophylaxis Campaign; Guyana, January-February 2005.” PLoS One 7 (7): e39672.

[40] Smith, J. K., Young, M. M., Wilson, K. L., and Craig, S. B. 2013. "Leptospirosis Following a Major Flood in Central Queensland, Australia.” Epidemiology \& Infection 141 (3): 585-90.

[41] Bennett, R. M. 1993. "Decision Support Risk-assessment and Simulation Models of the Costs of Leptospirosis in Dairy Herds.” Agricultural Systems 43 (2): 115-32.

[42] Van der Kamp, A., Dijkhuizen, A. A., and Peterse, D. J. 1990. "A Simulation of Leptospirosis Control in Dutch Dairy Herds." Preventive Veterinary Medicine 9 (1): 9-26.

[43] Caley, P., and Ramsey, D. 2001. "Estimating Disease Transmission in Wildlife, with Emphasis on Leptospirosis and Bovine Tuberculosis in Possums, and Effects of Fertility Control.” Journal of Applied Ecology 38 (6): 1362-70.

[44] Zaman, G., Khan, M. A., Islam, S., Chohan, M. I., and Jung, I. H. 2012. "Modeling Dynamical Interactions between Leptospirosis Infected Vector and Human Population.” Applied Mathematical Sciences 6 (26): 1287-302.

[45] Khan, M. A., Islam, S., Khan, S. A., Khan, I., Shafie, S., and Gul, T. 2014. "Prevention of Leptospirosis Infected Vector and Human Population by Multiple Control Variables.” Abstract and Applied Analysis 24: ID 619035.

[46] Schneider, M., Velasco-Hernandez, J., Min, K. D., Leonel, D., Baca-Carrasco, D., Gompper, M., ... and Munoz-Zanzi, C. 2017. "The Use of Chemoprophylaxis 
after Floods to Reduce the Occurrence and Impact of Leptospirosis Outbreaks." International Journal of Environmental Research and Public Health 14 (6): 1-18.

[47] Panaphut, T., Domrongkitchaiporn, S., and Thinkamrop, B. 2002. "Prognostic Factors of Death in Leptospirosis: A Prospective Cohort Study in Khon Kaen, Thailand.” International Journal of Infectious Diseases 6 (1): 52-9.

[48] Bonyah, E., Khan, M. A., Okosun, K. O., and Islam, S. 2017. “A Theoretical Model for Zika Virus Transmission." PloS one 12 (10): e0185540.

[49] Hill, D. S. 2008. Pests of Crops in Warmer Climates and Their Control. Dordrecht: Springer Science \& Business Media.

[50] Garba, B., Bahaman, A. R., Khairani-Bejo, S., Zakaria, Z., and Mutalib, A. R. 2017. "Retrospective Study of Leptospirosis in Malaysia.” EcoHealth 14 (2): 389-98.

[51] Singh, S. M. 2003. "Studies on Bio-ecology and Management of Indian Metad (Millardia meltada Gray) and Indian field Mouse (Mus booduga Gray).” Ph.D. thesis, The Chandra Shekhar Azad University of Agriculture and Technology, Kanpur, India.
[52] Aguirregabiria, J. M. Dynamics Solver [Online], 1992-2015, freeware, http://tp.lc.ehu.es/jma/ds/ds.html.

[53] Di Rienzo J. A., Casanoves, F., Balzarini, M. G., Gonzalez, L., Tablada, M., and Robledo, C. W. 2018. InfoStat versión 2018. Grupo InfoStat, FCA, Universidad Nacional de Córdoba, Argentina, http://www.infostat.com.ar.

[54] Bhardwaj, P., Kosambiya, J. K., and Desai, V. K. 2008. "A Case Control Study to Explore the Risk Factors for Acquisition of Leptospirosis in Surat City, after Flood.” Indian Journal of Medical Sciences 62 (11): 431-8.

[55] WHO (World Health Organization). 2011. "Leptospirosis: An Emerging Public Health Problem.” Weekly Epidemiological Record 86 (6): 45-50.

[56] Alderman, K., Turner, L. R., and Tong, S. 2012. "Floods and Human Health: A Systematic Review.” Environment International 47: 37-47.

[57] Shukla, J. B., Singh, V., and Misra, A. K. 2011. "Modeling the Spread of an Infectious Disease with Bacteria and Carriers in the Environment." Nonlinear Analysis: Real World Applications 12 (5): 2541-51. 